\title{
Barentsz, a New Component of the Staufen-Containing Ribonucleoprotein Particles in Mammalian Cells, Interacts with Staufen in an RNA-Dependent Manner
}

\author{
Paolo Macchi, ${ }^{1}$ Sven Kroening, ${ }^{1}$ Isabel M. Palacios, ${ }^{2}$ Simona Baldassa, ${ }^{1}$ Barbara Grunewald, ${ }^{1}$ Concetta Ambrosino, ${ }^{1}$ \\ Bernhard Goetze, ${ }^{1}$ Andrei Lupas, ${ }^{1}$ Daniel St. Johnston, ${ }^{2}$ and Michael Kiebler ${ }^{1}$ \\ ${ }^{1}$ Max-Planck-Institute for Developmental Biology, D-72076 Tübingen, Germany, and ${ }^{2}$ Wellcome Trust Cancer Research Institute, Department of Genetics, \\ University of Cambridge, Cambridge CB2 1QR, United Kingdom
}

\begin{abstract}
Staufen1, the mammalian homolog of Drosophila Staufen, assembles into ribonucleoprotein particles (RNPs), which are thought to transport and localize RNA into dendrites of mature hippocampal neurons. We therefore investigated whether additional components of the RNA localization complex besides Staufen are conserved. One candidate is the mammalian homolog of Drosophila Barentsz (Btz), which is essential for the localization of oskar mRNA to the posterior pole of the Drosophila oocyte and is a component of the oskar RNA localization complex along with Staufen. In this study, we report the characterization of mammalian Btz, which behaves like a nucleocytoplasmic shuttling protein. When expressed in the Drosophila egg chamber, mammalian Btz is still able to interact with Drosophila Staufen and reach the posterior pole in the wild-type oocyte, but does not rescue the btz mutant phenotype. Most interestingly, we show by immunoprecipitation assays that Btz interacts with mammalian Staufen in an RNA-dependent manner through a conserved domain, which encompasses the region of homology to the Drosophila Btz protein and contains a novel conserved motif. One candidate for an RNA that mediates this interaction is the dendritically localized brain cytoplasmic 1 transcript. In addition, Btz and Staufen 1 colocalize within particles in the cell body and, to a more variable extent, in dendrites of mature hippocampal neurons. Together, our data suggest that the mRNA transport machinery is conserved during evolution, and that mammalian Btz is an additional component of the dendritic RNPs in hippocampal neurons.
\end{abstract}

Key words: MLN51; Barentsz; Staufen; ribonucleoprotein particles; RNA transport; hippocampal neurons; BC1 RNA

\section{Introduction}

The asymmetric distribution and localization of mRNA have been described in many organisms and different cell types (Bashirullah et al., 1998; Bassell et al., 1999; Palacios and St. Johnston, 2001). For example, in Drosophila melanogaster, the localization of oskar and bicoid mRNAs is required to establish the embryonic body axes. In neurons, translation of dendritically localized mRNAs might be involved in mechanisms such as synaptic plasticity and memory (Kiebler and DesGroseillers, 2000; Smith et al., 2001; Steward and Schuman, 2001). Drosophila mutants in kinesin heavy chain, mago nashi, staufen, cytoplasmic tropomyosin II, $Y 14$, and barentsz (btz1) are all defective in the posterior localization of oskar mRNA (Ephrussi et al., 1991; Erdelyi et al., 1991; Newmark and Boswell, 1994; Kim-Ha et al., 1995; Tetzlaff et al.,

\footnotetext{
Received Jan. 9, 2003; revised April 18, 2003; accepted April 18, 2003.

This work was supported by PhD fellowships from the Italian government (S.B.) and the Claussen-SimonStiftung (S.K.), a Royal Society Dorothy Hodgkin postdoctoral fellowship (I.M.P.), the Sonderforschungsbereich SFB446 (P.M. and M.K.), and the Human Frontier Science Programme network grant (D.S.J. and M.K.). We thank Drs. L. DesGroseillers, M. Mallardo, and F. van Eeden for their critical comments.

Correspondence should be addressed to Dr. Michael Kiebler, Max-Planck-Institute for Developmental Biology, Spemannstrasse 35, D-72076 Tübingen, Germany. E-mail: michael.kiebler@tuebingen.mpg.de.

S. Baldassa's present address: Università degli Studi di Milano, Dipartimento di Fisiologia e Biochimica Generali, Milano, Italy.

Copyright $\odot 2003$ Society for Neuroscience $\quad$ 0270-6474/03/235778-11\$15.00/0
}

1996; van Eeden et al., 2001). The clearest link between any of these proteins and their effects on the localization of oskar mRNA has been in the case of Staufen protein. The double-stranded RNA binding protein Staufen is essential for the cytoplasmic localization of oskar mRNA and colocalizes with the oskar transcript to the posterior pole of the oocyte. In Drosophila, oskar mRNA assembles into ribonucleoprotein particles (RNPs) together with Mago nashi, Y14, Barentsz (Btz), and Staufen proteins (van Eeden et al., 2001). Btz thereby transiently colocalizes with oskar mRNA and Staufen to the posterior pole. Until now, only a few components of such RNP complexes, which are involved in RNA transport and localization in mammalian cells and, in particular, primary neurons, have been identified (Krichevsky and Kosik, 2001; Ohashi et al., 2002). The mammalian homolog of Drosophila Staufen has been implicated in RNA localization into dendrites of hippocampal neurons (Kiebler et al., 1999; Köhrmann et al., 1999a; Tang et al., 2001), and the Staufen-containing RNPs are thought to be the active transport units for dendritically localized mRNAs (Köhrmann et al., 1999a; Mallardo et al., 2003; Roegiers, 2003). Recently, protein phosphatase 1 was identified as a Staufen1 (Stau1)-interacting protein; this suggested that both proteins might be recruited into the same RNPs (Monshausen et al., 2002). An attractive candidate for an additional component of such Staufen-containing RNPs is the mammalian homolog of 
Drosophila Btz (van Eeden et al., 2001), which shares restricted homology with the human nucleocytoplasmic shuttling protein malignant lymph node 51 (MLN51) (Tomasetto et al., 1995; Degot et al., 2002). We show in this study that mammalian Btz (mBtz) does not rescue the $b t z 1$ mutant phenotype when exogenously expressed in the Drosophila oocyte. mBtz maintains the capacity, however, to interact with Drosophila Staufen and reach the posterior pole in the wild-type oocyte, suggesting a conserved function of this $\mathrm{mBtz}-$ Staufen complex. In hippocampal neurons, overexpressed mBtzand Staufen-tagged proteins colocalize in granular structures in the dendritic compartment. Whereas the degree of colocalization is highest within the cell body, it gradually decreases with progressive distance from the cell body toward distal dendrites. Most importantly, immunoprecipitation (IP) assays reveal that this interaction is RNA dependent. Therefore, $\mathrm{mBtz}$ may represent an important component of the dendritic mRNA transport particles in hippocampal neurons.

\section{Materials and Methods}

Animals. For cell culture, pregnant Sprague Dawley rats were used. For tissue extracts, 4 - to 6-week-old Sprague Dawley rats $(<250 \mathrm{gm})$ were anesthetized with ether and decapitated in accordance with the state law. All steps were performed under strictly RNase-free conditions at $4^{\circ} \mathrm{C}$ or on ice.

Cloning. Using information on expressed sequence tags (ESTs) and 5 '-rapid amplification of cDNA ends, the cDNAs encoding both the rat and mouse homologs of MLN51-Btz were cloned and sequenced. The rat and mouse Btz cDNAs were cloned either in frame with the pd2EGFP-N1 (Clontech, Heidelberg, Germany) or in the same vector without enhanced green fluorescent protein (EGFP) to express untagged Btz protein.

For the expression of $\mathrm{mBtz}$ in Drosophila, the mBtz coding region was cloned into the BamHI-SpeI sites of pD277-green fluorescent protein (GFP)6 vector (Micklem et al., 1997; van Eeden et al., 2001) to generate a transformation construct in which the $\alpha 4$-tubulin promoter drives the germline-specific expression of an mBTZ-GFP6 fusion protein. The construct to express Staufen1-hemagglutinin (HA) was described previously by Duchaîne et al. (2000).

For $\mathrm{Btz}_{1-158}-\mathrm{GFP}, \mathrm{Btz}_{152-359}-\mathrm{GFP}$, and $\mathrm{Btz}_{1-359}-\mathrm{GFP}, \mathrm{cDNAs}$ from rat brain were amplified by PCR using the Herculase HotStart DNA polymerase (Stratagene, Heidelberg, Germany) with the following primers: 5'-GCTAGCATGGCGGACCGGCGGCGGC (NheI, 1), 5' ACCGGTTTGTTCTCCACAGGCTCTGTG (AgeI, reverse 158), 5' GCTAGCATGACAGAGCCTGTGGAGAACAAAG (NheI, 152), and 5'-ACCGGTTTAGCTTCATGCTTAAGAGTCTC (AgeI, reverse 359). (Underlining indicates the sequence recognized by the corresponding restriction enzymes.) The PCR products were sequenced and cloned in frame with the $\mathrm{d} 2 \mathrm{EGFP}$. The $\mathrm{L}_{465}$ A point mutation was created using the QuickChange site-directed mutagenesis kit (Stratagene) according to the manufacturer's protocol with the following primers: $5^{\prime}$-GAGCAAGATGTGGCACAGGCGAATATAGCAGAACAAAATTGGAGT and 5'-ACTCCAATTTTGTTCTGCTATATTCGCCTGTGCCACATCTTGCTC.

Computer analysis. Sequences related to mouse MLN51 were identified in the nonredundant database at National Center for Biotechnology Information using Blast (http://www.ncbi.nlm.nih.gov/BLAST/) and retrieved using Entrez (http://http://www.ncbi.nlm.nih.gov/Entrez/). The relationship between MLN51 and thyroid hormone receptorassociated protein $150 \mathrm{kDa}$ (TRAP150) sequences was detected through pattern searches at Pôle Bio-Informatique Lyonnais using Pattinprot [http://npsa-pbil.ibcp.fr/cgi-bin/npsa_automat.pl?page=/NPSA/npsa_ pattinprot.html]. Alignments were made in Macaw (Schuler et al., 1991).

Northern blotting and in situ hybridization. Northern analysis was performed using commercial mouse mRNA blots (Clontech). The cDNA encoding the full-length mouse Btz protein was labeled with $\left[\alpha-{ }^{32} \mathrm{P}\right]$ deoxycytidine triphosphate using Prime-It RmT random primer labeling kit (Stratagene); prehybridization and hybridization steps were performed according to the manufacturer's protocol (Clontech). The in situ hybridization was performed as described previously by Knoell et al. (2001). Antisense and sense RNA probes labeled with digoxigenin were synthesized according to the manufacturer's protocol (Roche, Mannheim, Germany). Postnatal day 14 (P14) mouse brains were snap-frozen, cryosectioned, and then fixed in 4\% paraformadehyde for $20 \mathrm{~min}$.

Antibody production. Amino acids 175-317 and 356-527 of the rat Btz gene and amino acids 109-353 from the mouse Btz gene were expressed in Escherichia coli using the pPROEX-T vector (Invitrogen, Karlsruhe, Germany). The first fusion protein was purified under denaturing conditions, and the others were purified under native conditions using nickel-nitrilotriacetic acid chromatography (Qiagen, Hilden, Germany). For immunization in rabbits, the first two antigens were used, whereas the third one was injected into mice. Antigen 2 was then used for affinity purification.

In vitro transcription-translation. The cDNA of mBtz cloned in Bluescript KS (Stratagene) was transcribed and translated using the TNT quick-coupled transcription-translation system (Promega, Mannheim, Germany) according to the manufacturer's instructions in the presence of $\left[{ }^{35} \mathrm{~S}\right]$ methionine. Samples were run on SDS-polyacrylamide gels and then exposed for $1-6 \mathrm{hr}$.

Immunocytochemistry. Immunocytochemistry on mature primary hippocampal neurons in culture [16 $\mathrm{d}$ in vitro (DIV)] was performed as described previously (Duchaîne et al., 2002). The following primary antibodies (incubated overnight at $4^{\circ} \mathrm{C}$ ) were used: affinity-purified polyclonal rabbit anti-Btz (dilution, 1:50) and mouse polyclonal anti-Staufen 1 antibodies (1:200). As secondary antibodies, Alexa Fluor 488 goat antirabbit IgG [heavy and light $(\mathrm{H}+\mathrm{L})$ ] conjugated (1:500; Molecular Probes, Leiden, The Netherlands) and Cy3-conjugated goat anti-mouse IgG (H+L) (1:500; Dianova, Hamburg, Germany) were used. Fluorescence and confocal microscopy were performed as described previously (Kiebler et al., 1999; Macchi et al., 2003). The Metamorph 5.0 software package was used to quantify the degree of colocalization between $\mathrm{mBtz}$ and Staufen 1 in hippocampal neurons. Confocal pictures of hippocampal neurons immunostained with anti-mBtz and anti-Staul polyclonal antibodies were analyzed in detail by choosing random areas from three different compartments (cell body, proximal dendrite, or distal dendrite), and the percentage of colocalization ( $\mathrm{mBtz}$ vs Staufen 1 ) was measured. The mean and SD were then calculated using Microsoft Excel software.

Cell culture, transient transfection, and LMB treatment. Baby hamster kidney (BHK) cells were grown and transfected as described previously by Macchi et al. (2003). For leptomycin B (LMB) experiments, BHK cells were treated ( $16 \mathrm{hr}$ after transfection) with $50 \mathrm{~nm}$ LMB for $3 \mathrm{hr}$ before fixation. Adult primary hippocampal neurons were transfected as described previously by Köhrmann et al. (1999b). Cells were analyzed by fluorescence microscopy as described previously by Köhrmann et al. (1999a).

Fly stocks and Staufen antibody stainings. The barentsz mutant allele used was w;e btz ${ }^{1} c a / T M 3 S b$ (van Eeden et al., 2001). The mBtz-GFP construct was introduced into flies using standard methods. The transgenic used was $w ; m B t z G F P / C y O$. Antibody stainings were performed as described previously by Palacios and St. Johnston (2002), and the antiStaufen antibody was used at 1:1000 (St. Johnston et al., 1991).

Preparation of cell and tissue extracts. BHK cells were washed with PBS, lysed in lysis buffer [0.1\% Triton X-100, $150 \mathrm{~mm} \mathrm{KCl,} 50 \mathrm{~mm}$ HEPES, $\mathrm{pH}$ 7.4 , and $1 \mathrm{~mm}$ DTT plus proteinase inhibitor mixture (Roche), including $1 \mathrm{~mm}$ phenylmethylsulfonyl fluoride (PMSF)]. Extracts $(50 \mu \mathrm{g})$ from brain tissue as well as extracts from cultured glia or hippocampal neurons were prepared. Equal amounts of proteins were separated by $8 \%$ SDSPAGE and blotted onto nitrocellulose (Schleicher \& Schuell, Dassel, Germany). Western blot analysis was performed as described previously by Macchi et al. (2003).

For both the DNase treatment and the RNase treatment, two rat forebrains were homogenized in a $5 \mathrm{ml}$ Dounce homogenizer on ice with $4 \mathrm{ml}$ of lysis buffer using 10 manual strokes, followed by 10 strokes in a motordriven Dounce. The homogenate was centrifuged for $10 \mathrm{~min}$ at $21,000 \times$ $g$ [supernatant at $14,000 \times \mathrm{rpm}(\mathrm{S} 14)]$. For RNase treatment, $5 \mathrm{~mm}$ EDTA was added to the supernatant, and the sample was divided in half. One sample (+RNase, DNase free; Sigma, Munich, Germany) was incu- 
bated for $1 \mathrm{hr}$ at $30^{\circ} \mathrm{C}$ with $40 \mu \mathrm{g} / \mathrm{ml}$ RNase A in $10 \mathrm{~mm}$ Tris-Cl, $\mathrm{pH} 7.5$, and $15 \mathrm{~mm} \mathrm{NaCl}$, and the other one-half, as control, was incubated in an equal amount of buffer (-RNase). For DNase (MBI Fermentas, St. LeonRot, Germany) treatment, the S14 was incubated with DNase buffer alone (control) or together with $2 \mathrm{U}$ of DNase at $37^{\circ} \mathrm{C}$ for $30 \mathrm{~min}$, and after $15 \mathrm{~min}$, an additional $2 \mathrm{U}$ of the enzyme were added. After incubation, samples were centrifuged for $20 \mathrm{~min}$ at $100,000 \times g$ to collect the pellet at $100,000 \times g(\mathrm{P} 100)$ and supernatant at $100,000 \times g(\mathrm{~S} 100)$, respectively. The pellets were then processed as described previously by Mallardo et al. (2003).

Immunoprecipitations. BHK cells grown in $5 \mathrm{~cm}$ Petri dishes were transfected, washed in PBS at 14-18 hr posttransfection, and then harvested in $500 \mu \mathrm{l}$ of lysis buffer [0.5\% Triton X-100, $50 \mathrm{~mm}$ Tris-Cl, pH 7.5, $100 \mathrm{~mm} \mathrm{KCl,} 1 \mathrm{~mm}$ PMSF, and protease inhibitor mixture (Roche)]. The lysate was centrifuged at $14,000 \times g$ for $10 \mathrm{~min}$ at $4^{\circ} \mathrm{C}$. For immunoprecipitations, $500 \mu \mathrm{g}$ of total protein were preincubated in a total volume of $1 \mathrm{ml}$, either with anti-HA ascites fluid (1:333) or affinitypurified rabbit polyclonal anti-GFP antibodies (1:500, Molecular Probes) and $30 \mu \mathrm{l}$ of $50 \%$ protein A-Sepharose slurry (Amersham Biosciences, Freiburg, Germany) equilibrated in lysis buffer for at least $1 \mathrm{hr}$ at $4^{\circ} \mathrm{C}$ and then centrifuged at $500 \times g$ for $10 \mathrm{sec}$ at $4^{\circ} \mathrm{C}$. The pellet was washed four times with washing buffer $(0.1 \%$ Triton X-100, 50 mM Tris$\mathrm{Cl}, \mathrm{pH}$ 7.5, $100 \mathrm{~mm} \mathrm{KCl}$, and $1 \mathrm{~mm}$ PMSF), resuspended in reducing (DTT-containing) sample buffer, and analyzed by SDS-PAGE and Western blotting using polyclonal rabbit anti-GFP (1:2000; Molecular Probes), monoclonal mouse anti-GFP (1:2000; Clontech) or monoclonal anti-HA (1:1000; Roche) antibodies. The RNase treatment was performed as described previously. For the immunoprecipitations of the endogenous complexes, embryonic day 17 rat forebrains were lysed in lysis buffer. The preclearing was performed by incubation of the lysate ( 2 $\mathrm{mg}$ of protein in total in each sample) with preimmune serum and protein $\mathrm{A}$ for $1 \mathrm{hr}$ at $4^{\circ} \mathrm{C}$. After centrifugation, the supernatants were immunoprecipitated with the indicated antibodies and protein A. The immunocomplexes were collected by centrifugation and washed. Samples were then processed as described above.

RNA isolation and radioactive reverse transcription (RT)-PCR from immunoprecipitates were performed as described previously by Mallardo et al. (2003) with the following modifications. $\mathrm{MgCl}_{2}$ (1 mM) was added to both the lysis and the washing buffer. The latter also contained $0.5 \%$ Triton X-100 (instead of $0.1 \%$ ). To avoid a possible DNA contamination, extracts were incubated with RNase-free DNase (MBI Fermentas). First-strand cDNA synthesis was performed with random hexamers by using Moloney murine leukemia virus reverse transcriptase (Genaxxon, Stafflangen, Germany). A semiquantitative PCR was then achieved using the following primers: 5 '-GGGGTTGGGGATTTAGCTC (forward) and 5'-GGTTGTGTGTGCCAGTTACC (reverse) for brain cytoplasmic 1 (BC1); 5' -CATGCCCCCATTCCATCTG (forward) and 5'-GGACCCACTCCACAAACTC (reverse) for microtuble-associated protein 2 (MAP2); TTCGCGGGCGACGATGCTCC (forward) and $5^{\prime}$-CAGGTCCAGACGCAGGATGG (reverse) for $\beta$-actin; and 5'-CTCCGGTTAAATGGGAGTGG and5'-CCCGGCACTAGTTACACTTG (reverse) for Histone H1.

\section{Results}

A recent study identified a novel protein called Barentsz (Btz), which is involved in the posterior localization of oskar mRNA in Drosophila and is a component of the oskar RNA transport machinery together with Staufen protein (van Eeden et al., 2001). In an attempt to further characterize the function and composition of the Staufen-containing RNPs in vertebrate neurons (Köhrmann et al., 1999a; Kiebler and DesGroseillers, 2000; Krichevsky and Kosik, 2001; Ohashi et al., 2002; Mallardo et al., 2003), we examined whether a mammalian homolog of Drosophila Barentsz (dBtz) has been conserved during evolution. The $\mathrm{N}$-terminal part of $\mathrm{dBtz}$ protein showed significant homology to the human MLN51 protein whose mRNA is overexpressed in breast cancer cell lines (Tomasetto et al., 1995) and in gastric cancer (Varis et al., 2002). Using the EST data bank, we cloned the

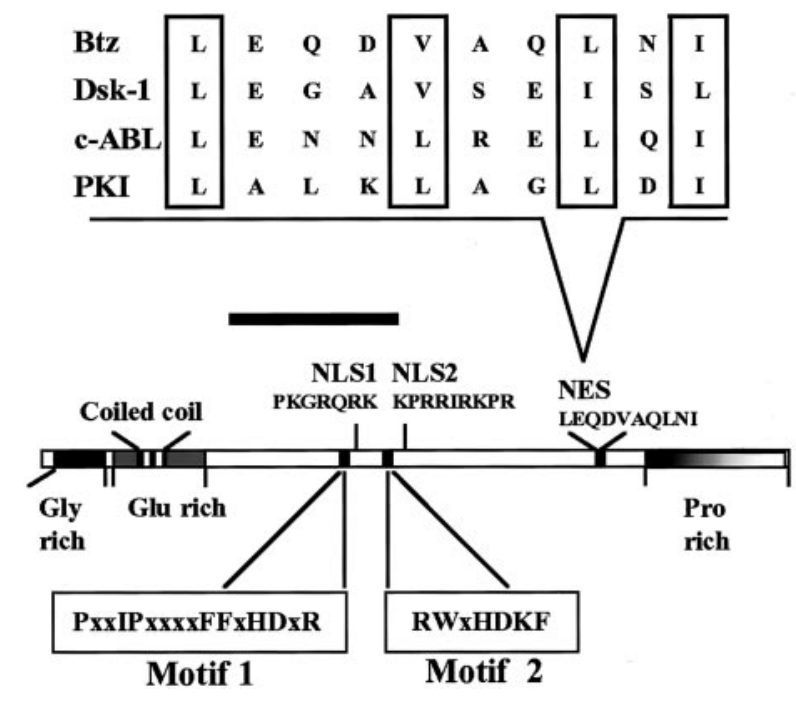

Figure 1. Domain structure of mammalian Barentsz. Schematic representation of rat Btz protein. The different domains are indicated. Two putative NLSs and an NES have been predicted. The black bar indicates the region of homology to Drosophila Btz. A comparison of the NES sequences of $\mathrm{mBtz}$, drosulfakinin (Dsk1), Abelson murine leukemia viral oncogene (c-ABL), and protein kinase inhibitor (PKI) is shown on the top of the figure. Hydrophobic residues important for NES activity are indicated by open boxes.

full-length mouse (accession number AF526276) and rat (accession number AF525467) homologs of dBtz. The mouse Btz transcript shows an open reading frame of 2094 nucleotides that encodes a protein of 698 aa and a predicted molecular mass of $\sim 76$ $\mathrm{kDa}$. The rat Btz protein consists of 699 aa and shares $97 \%$ of identity with its mouse homolog. A schematic representation of the rat Btz protein is given in Figure 1. The protein contains a glycine-rich region, a glutamine-rich region, a coiled-coil domain at the $\mathrm{N}$ terminal and a proline-rich region at the $\mathrm{C}$ terminal as in Drosophila. Four putative Src homology $(\mathrm{SH}) 3$ domain binding sites, one putative $\mathrm{SH} 2$ domain binding site, and two potential monopartite nuclear localization signals (NLSs) were predicted by Degot et al. (2002). In addition, one nuclear export signal (NES) consensus sequence is present at the $\mathrm{C}$ terminal (Fig. 1). Interestingly, one consensus sequence for the NLS appears to be conserved in Drosophila. However, the functionality of the NLS within dBtz has not yet been experimentally verified. The $\mathrm{NES}$, in contrast, is present only in mBtz. Additionally, a thorough computer analysis revealed the existence of two conserved motifs that are present in the N terminal of MLN51 proteins and at the very C-terminal end of TRAP150 proteins (Fig. 1, supplementary data; available at www.jneurosci.org). No additional conserved domains were identified.

\section{Expression of $\mathrm{mBtz}$ in different tissues}

The expression of the mammalian $B t z$ transcript was then tested by Northern blotting of different mouse tissues (Fig. $2 A$ ). A single transcript of $\sim 4.4 \mathrm{~kb}$ was found to be expressed at a high level in heart, brain, liver, kidney, and testis. Lower expression was detected in muscle, lung, and spleen. This fits well with the identification of a single $4.2 \mathrm{~kb}$ transcript of MLN51 in all of the breast cancer cell lines, many other human cell lines, and a wide variety of human tissues (Degot et al., 2002). Because we were interested in the expression of $\mathrm{mBtz}$ in the hippocampus, we performed in situ hybridization on mouse brain sections (Fig. 2 B). Btz mRNA was detected in the hippocampus, in particular in the cell body layers of the dentate gyrus and the CA1 and CA3 regions (Fig. 
A

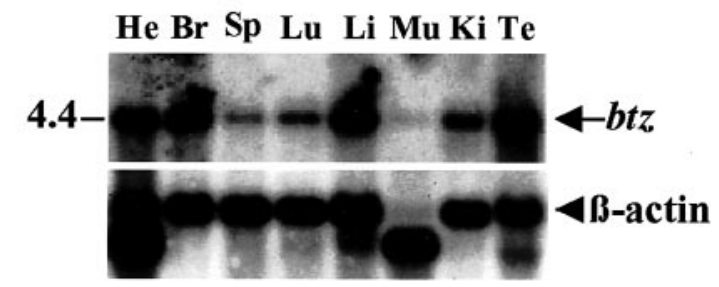

B
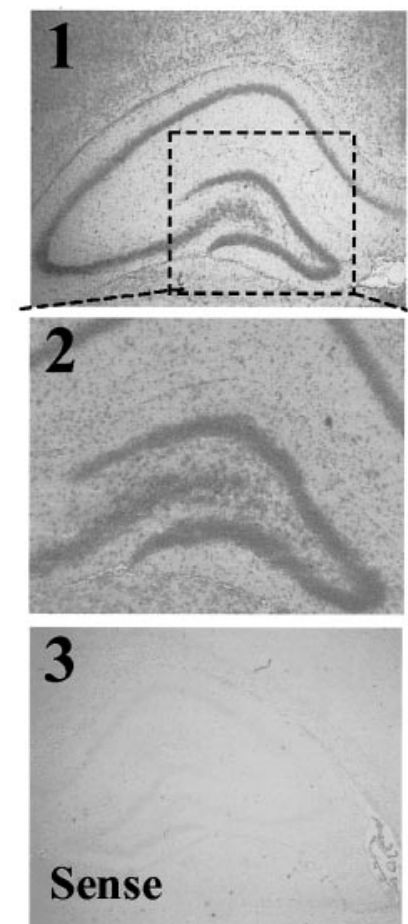

Figure 2. Expression of $B t z m R N A$ in different tissues. $A$, Northern blot analysis of polyA mRNA from the following different mouse tissues: heart (He), brain (Br), spleen (Sp), lung (Lu), liver (Li), skeletal muscle (Mu), kidney (Ki), and testis (Te). Bottom panel, $\beta$-Actin control. B, Btz mRNA is expressed in the hippocampus. In situ hybridization on P14 mouse brain sections showing the expression of Btz mRNA. Coronal section of the hippocampus (1), including a high magnification insert (2). 3, In situ hybridization performed with the sense probe in the hippocampus.

$2 B 1,2)$. Btz mRNA was also expressed in other regions of the brain, especially in the cerebellum (data not shown). No signal was detected in the hippocampus when the sense probe was used instead (Fig. 2B3).

\section{$\mathrm{mBtz}$ is a nucleocytoplasmic shuttling protein}

To determine the subcellular localization of Btz, we cloned the full-length cDNA of mouse Btz in front of the green fluorescent protein reporter $(\mathrm{mBtz}-\mathrm{GFP})$. When the resulting construct was transfected into BHK, mBtz-GFP was almost excluded from the nucleus and showed a prominent cytoplasmic distribution, which was often concentrated at the perinuclear region (Fig. $3 A$, left panel, arrow). A similar expression pattern was observed when yellow fluorescent protein was cloned at the $\mathrm{N}$ terminus instead and expressed in BHK cells to exclude the possibility that the position of the added tag influences the intracellular localization of the fusion protein (data not shown). Because the Btz protein contains both predicted NLS and NES domains, we wanted to test whether Btz enters the nucleus or not. LMB specifically inhibits chromosome region maintenance 1 (CRM1)dependent protein export from the nucleus (Fukuda et al., 1997). LMB treatment led to the accumulation of Btz-GFP in the nucleus (Fig. $3 A$, right panel). The same results (Fig. $3 A$, bottom panels) were obtained using axin 1 as a positive control, a CRM1dependent nucleocytoplasmic shuttling protein (F. Fagotto, unpublished observations). These results suggested that both the predicted NLS and the NES in mammalian Btz are indeed functional. To further test this hypothesis, a series of deletion constructs were expressed in BHK cells, and their intracellular localization was analyzed. In contrast to wild-type Btz (Fig. 3B, row i), a truncated Btz-GFP fusion protein that contains the two putative NLSs but lacks the putative NES accumulated within the nucleus (Fig. 3B, row ii). To further map the region of Btz required for nuclear import, we fused GFP to a series of N-terminal fragments. $\mathrm{Btz}_{1-158}-\mathrm{GFP}$ was not imported into the nucleus (Fig. $3 B$, row iii); the $\mathrm{Btz}_{152-359}-\mathrm{GFP}$, in contrast, ended up exclusively in the nucleus (Fig. $3 B$, row iv). These results indicate that the two NLSs are functional within Btz. In addition, a signal appears to exist within the $\mathrm{N}$-terminal first 159 aa that could potentially interact with an unknown factor in the cytoplasm partially overriding the exclusive nuclear localization of $\mathrm{Btz}_{152-359}-$ GFP; this is currently under investigation.

To further investigate whether the putative NES is indeed functional, we introduced a point mutation within the NES consensus region $\left(\mathrm{L}_{458} \mathrm{XxxV}_{462} \mathrm{XxL}_{465} \mathrm{xI}_{467}\right)$ by changing the indicated leucine 465 (Fig. 1) to alanine. The mutant $\mathrm{Btz}_{\mathrm{L} 465 \mathrm{~A}}-\mathrm{GFP}$ now accumulated in the nucleus because of the abolished nuclear export (Fig. $3 B$, row v). To verify that Btz is behaving as a nucleocytoplasmic shuttling protein in nerve cells also, we transiently transfected hippocampal neurons (Köhrmann et al. 1999b) with constructs expressing either $\mathrm{mBtz}-\mathrm{GFP}$ or $\mathrm{N}$-terminal portions of mBtz tagged with GFP (Fig. $3 C$ ). Neurons expressing mBtzGFP showed perinuclear and cytoplasmic staining (Fig. 3C, first panel); signals were also detected in dendrites as discrete punctate structures when images were acquired at high magnification and increased exposure time (Fig. 3C, top inset). When neurons were transfected with the domain of $\mathrm{mBtz}$ containing the two NLSs $\left(\mathrm{Btz}_{152-359}-\mathrm{GFP}\right)$, the recombinant protein accumulated in the nucleus (Fig. 3C, fourth panel). The expression of the $\mathrm{N}$-terminal half of Btz $\left(\mathrm{Btz}_{1-359}\right)$ led to both cytoplasmic and nuclear localization (Fig. $3 C$, second panel); in addition, the expression of $\mathrm{Btz}_{1-158}-\mathrm{GFP}$ caused cytoplasmic localization of the recombinant protein (Fig. $3 C$, third panel). The same results were observed in transfected glial cells (small insets on the right). Together, these experiments indicate that both the NLS and the NES sequences in Btz are functional and that $\mathrm{Btz}$ is a nucleocytoplasmic shuttling protein in mammalian cells.

\section{mBtz interacts with Staufen in the Drosophila oocyte}

To test whether mBtz is a functional homolog of the Drosophila protein, we analyzed whether expression of $\mathrm{mBtz}$ fused to GFP (mBtz-GFP) in btz1 mutant egg chambers rescued the phenotype. In most $b t z 1$ homozygous egg chambers, both Staufen protein and oskar mRNA failed to localize to the posterior pole of the oocyte at stage 9 and remained at the anterior pole instead, until the beginning of stage 10B. However, occasionally, some oskar mRNA may localize to the posterior pole at stage 9 and become anchored there throughout oogenesis (van Eeden et al., 2001). When mBtz-GFP was expressed in wild-type chambers, its local- 
ization was indistinguishable from the endogenous dBtz protein (Fig. $4 A$ ). mBtzGFP accumulated in the oocytes in the germarium and to the posterior crescent at stage 5 (Fig. $4 A$, left panel). Furthermore, $\mathrm{mBtz}$ also concentrated around the nuclei of the nurse cells (Fig. $4 A$, middle panel). Finally, mBtz localized to the posterior pole of the stage 9 oocyte when oskar mRNA is transported to this pole (Fig. $4 A$, right panel, arrow; $B$, top panel). In heterozygous flies, $\mathrm{mBtz}$ was also detected at the posterior pole, most likely in a complex with Staufen (Fig. $4 B$, top panels). In the btzl background (Fig. $4 B$, middle panels), however, mBtz and Staufen did not localize to the posterior pole and were instead found at the anterior pole of the oocyte. The complex of mouse Btz-Stau and probably oskar mRNA entered the oocyte (they did not stay in the nurse cells), but the final anterior to posterior transport was blocked. Together, all of these results show that mBtz is able to interact in vivo with Drosophila Staufen and is a component of the oskar mRNA complex but is not able to function in the transport of oskar mRNA to the posterior pole of the oocyte.

Btz is present in Staufen 1 particles in dendrites of hippocampal neurons

To further investigate the role of mammalian Btz, we raised polyclonal antisera against several domains of the protein in rabbits and mice and tested its expression level in the brain. On Western blots of rat brain extract, the antibodies specifically recognized a band of $\sim 115 \mathrm{kDa}$ (Fig. $5 A$, left panel, I), higher then the predicted molecular mass of $76 \mathrm{kDa}$. This abnormal electrophoretic mobility, however, had also been observed for Drosophila Btz (van Eeden et al., 2001) as well as for human MLN51 protein (Degot et al., 2002). The preimmune serum, in contrast, gave no specific bands (Fig. 5A, left panel, PI). We then went on to affinity-purify the Btz antisera. As indicated in the right lane of Figure $5 A$ (Pur.), these antibodies were monospecific for the $115 \mathrm{kDa}$ band. A second higher band was detected (Fig. 5A, arrowhead); this corresponds to a phosphorylated form of Btz, because the treatment with alkaline phosphatase abolished this band (data not shown). To exclude possible posttranslational modifications of the Btz protein, we performed an in vitro translation and subsequent SDSPAGE (Fig. 5A, right panel). A single band was observed at $115 \mathrm{kDa}$, proving that the antibodies specifically recognize fulllength Btz protein. Furthermore, on
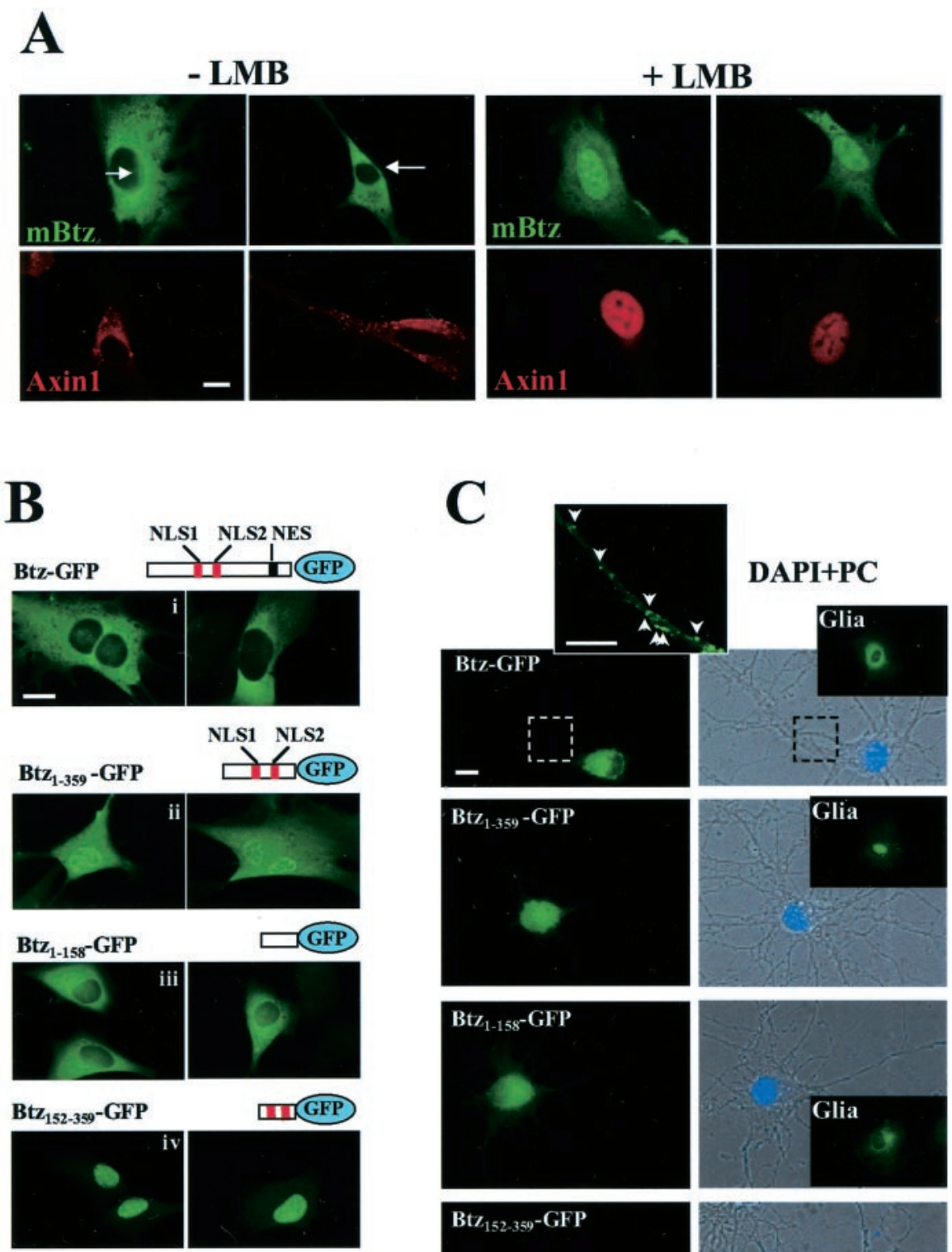

LEQDVAQANI
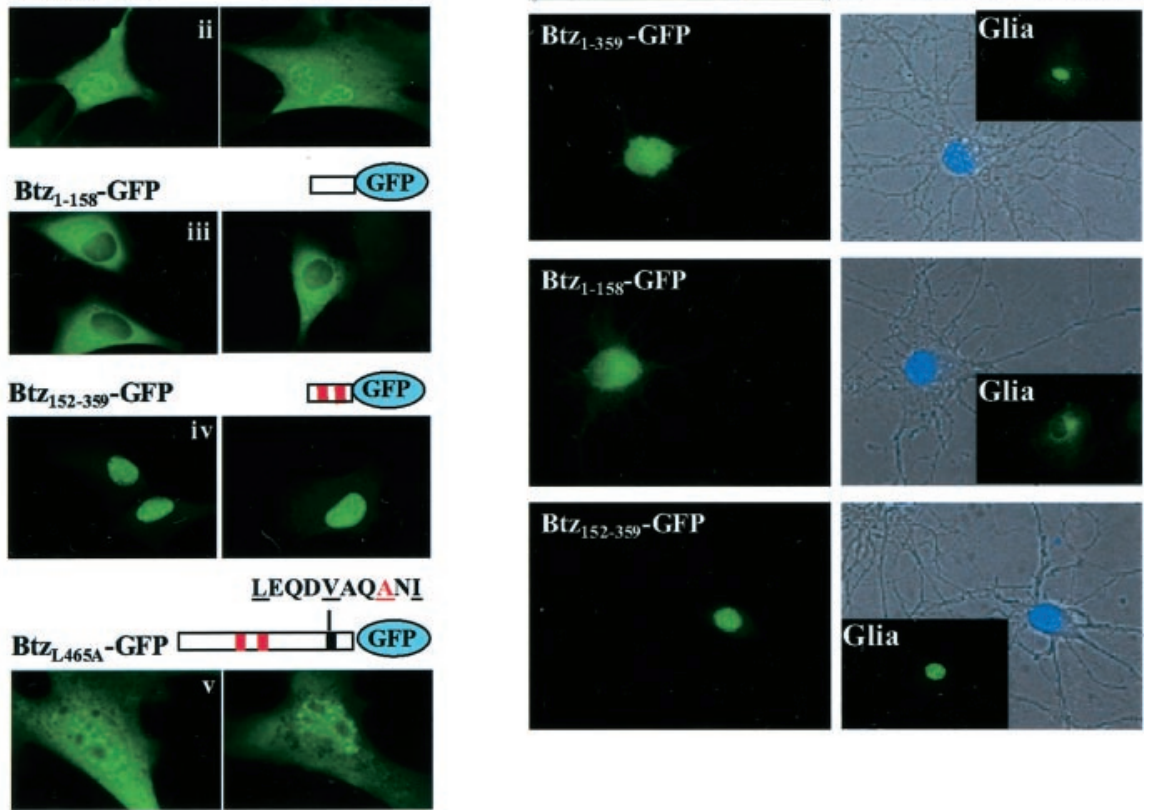

Figure 3. Leptomycin B-sensitive export of $\mathrm{mBtz}$ protein. $A, \mathrm{LMB}$ treatment of $\mathrm{BHK}$ cells expressing $\mathrm{mBtz}-\mathrm{GFP}$ fusion protein (mBtz-GFP). The CRM1-dependent axin1 served as control (F. Fagotto, unpublished observations). Three hour treatment with LMB causes the retention of Btz protein within the nucleus. Arrows indicate the perinuclear and cytoplasmic pattern of Btz-GFP. The experiments were performed either in the absence ( $-L M B)$ or in the presence (+LMB) of LMB. Scale bar, $10 \mu \mathrm{m} . B$, Mapping of the nuclear localization and nuclear export signal of mammalian Btz. BHK cells transfected with Btz-GFP show cytoplasmic localization of the fusion protein ( $i)$. In the subsequent panels, cells were transfected with a vector expressing the N-terminal half

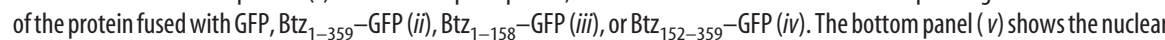
accumulation of $\mathrm{Btz}_{\mathrm{L465A}}-\mathrm{GFP}$, in which leucine 465 of the putative NES has been mutated to alanine. Scale bar, $10 \mu \mathrm{m}$ (for all images in $B)$. C, Expression of Btz-GFP in transiently transfected hippocampal neurons confirms the phenotype observed in transfected BHK cells. GFP fluorescence is shown on the left, whereas both the respective $4^{\prime}, 6^{\prime}$-diamidino-2-phenylindole (DAPI) staining and phase contrast $(\mathrm{PC})$ are shown on the right. Transfected glia are shown in the small panels. Arrowheads indicate the particulate Btz-GFP structures observed in dendrites. The inset represents a high magnification with increased exposure time of the area indicated by the dotted square. Scale bar, $10 \mu \mathrm{m}$ (for all images in C). 
A

\section{mBtz-GFP}
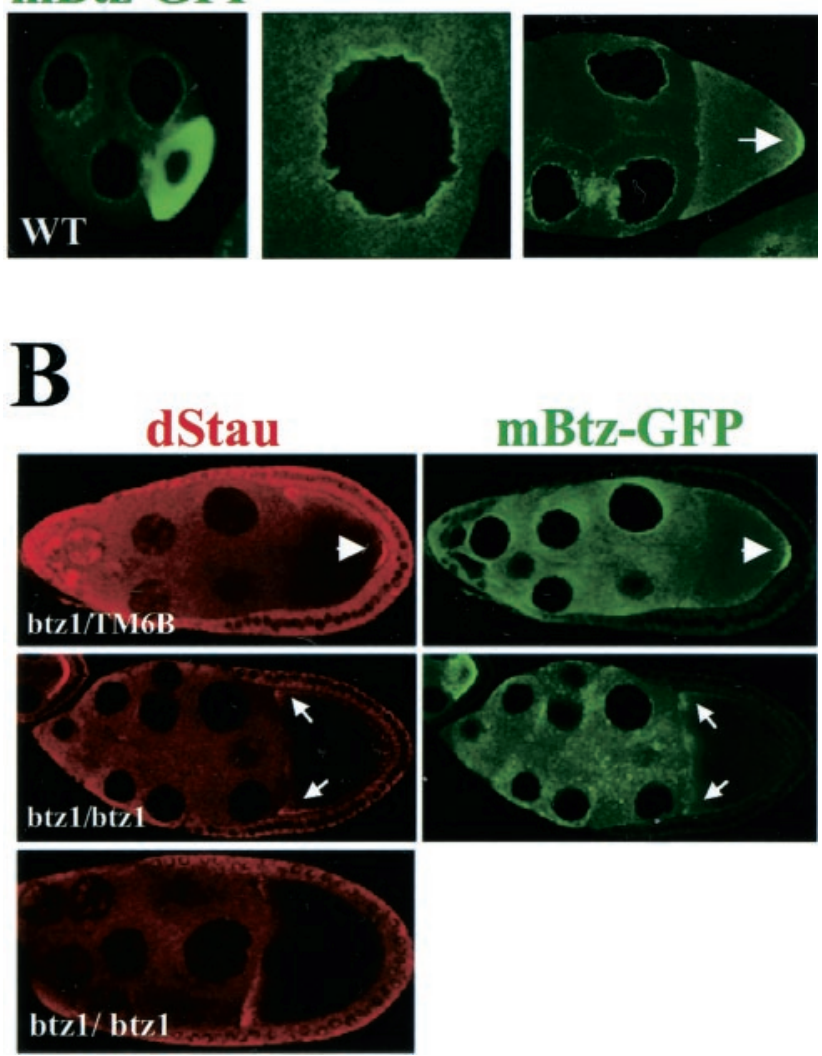

Figure 4. Mammalian Btz colocalizes with Staufen and oskar mRNA in the Drosophila oocyte. A, Localization of mBtz-GFP in the wild-type (WT) Drosophila germ line. Similar to Drosophila Btz, $\mathrm{mBtz}$ accumulates in the stage 5 oocyte (left panel), in a rim around the nuclei of the nurse cells (middle panel), and at the posterior pole of the stage 9 oocyte (right panel, arrow), where both Staufen and oskar mRNA localize. B, Localization of Drosophila Staufen (dStau) (red) and mBtz-GFP (green) in btz1/TM6B and btz1 mutant egg chambers expressing mBtz-GFP (top and middle panels, respectively). The arrowheads indicate the colocalization of Drosophila Staufen and mBtz-GFP proteins to the posterior pole. The bottom panel shows Staufen localization in $b t z 1$ mutant egg chambers that do not express mBtz-GFP. In the btz 1 mutant 0ocyte, both Staufen and $\mathrm{mBtz}-$ GFP do not accumulate at the posterior pole but instead localize at the anterior pole (middle panel, arrows). Thus, mBtz-GFP is unable to rescue the oskar mRNA localization phenotype in barentsz mutant egg chambers.

Western blots from BHK cell extracts and different neuronal tissue samples (Fig. 5B), the antibodies recognized in all of the cases a protein with the same molecular mass that has the same electrophoretic mobility as the untagged, full-length Btz protein overexpressed in BHK cells (Fig. 5B, right panel). Together, these experiments indicate that the antibodies specifically identify the $115 \mathrm{kDa}$ protein as $\mathrm{mBtz}$ protein. These results encouraged us to investigate whether $\mathrm{mBtz}$ and Staufen also interact in mammalian cells. As an experimental system, we chose isolated hippocampal neurons in culture in which Staufen is expressed in RNA-containing particles in the somatodendritic domain (Kiebler et al., 1999). Immunocytochemistry was performed on mature hippocampal neurons using anti-mBtz antibodies. When we focused on the cell body of these cells (Fig. 6A), Btz is predominantly found in the cytoplasm with a strong perinuclear and granular localization pattern (Fig. $6 \mathrm{~A}$, top panel). The localization of the endogenous Btz protein was then compared with the expression pattern of Btz-GFP in transfected hippocampal neu-

\section{A}
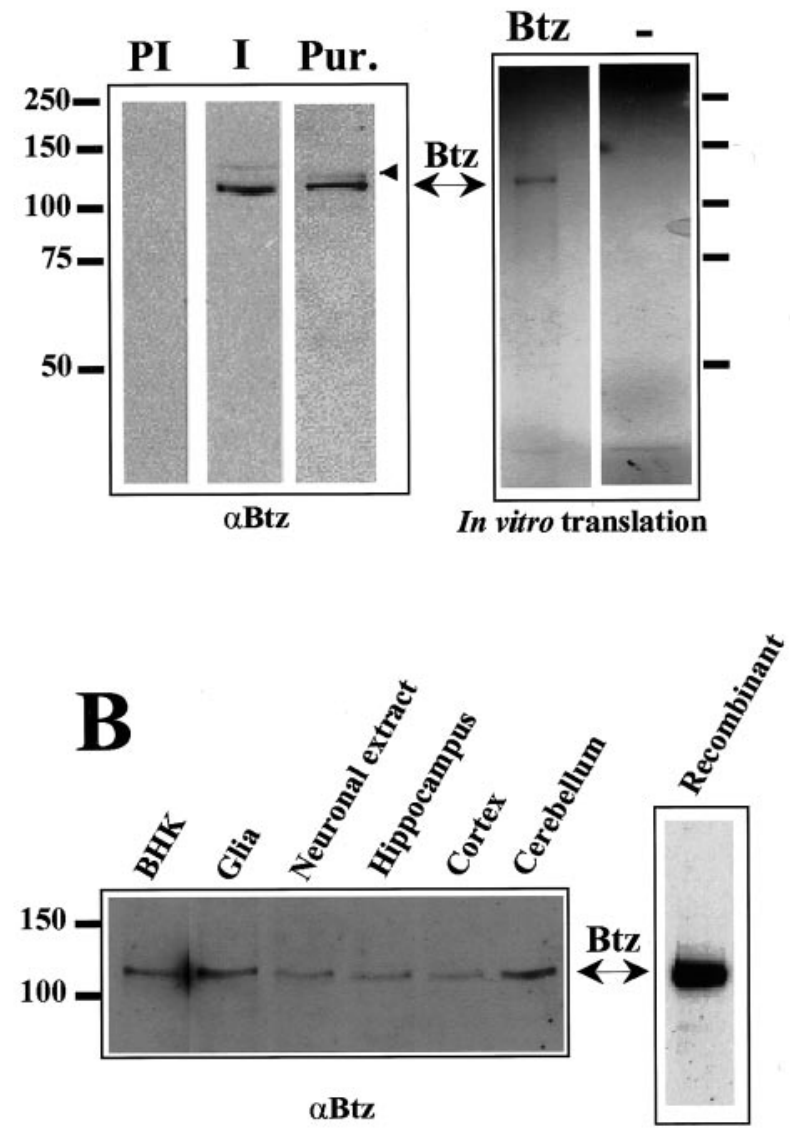

Figure 5. Mammalian Btz is a $115 \mathrm{kDa}$ protein. $A$, Western blot of extracts from hippocampal neurons probed with either preimmune (PI) (lane 1) or the corresponding immune antisera. Lane 2, A monospecific mouse polyclonal antisera (I); lane 3, affinity-purified (Pur.), rabbit polyclonal anti-Btz. Our different monospecific polyclonal antisera recognize a $115 \mathrm{kDa}$ protein; this is in good accordance with the observed molecular mass of Drosophila Btz, which runs as a $125 \mathrm{kDa}$ protein. Right panel, In vitro translation experiment showing that $\mathrm{mBtz}$ protein migrates as a $115 \mathrm{kDa}$ protein. A negative control ( - ) was performed without DNA. The arrowhead identifies the phosphorylated form of $\mathrm{mBtz}$. $B$, Western blot using extracts of different tissues. The antibodies recognize the protein overexpressed in BHK (right panel) but also the endogenous protein from BHK or from different neuronal tissues (left panel). $\alpha \mathrm{Btz}$, Anti-Btz antibodies.

rons. Both patterns were remarkably similar (Fig. 6A, bottom panel). In particular, $\mathrm{mBtz}-\mathrm{GFP}$ formed granules in the perinuclear region (Fig. 6A, inset), indicating that our reporter protein actually behaved like the endogenous counterpart. Similar results were obtained by transfecting hippocampal neurons with constructs expressing untagged mBtz protein (data not shown). Confocal microscopy showed that Staufen 1 and mBtz colocalized in the perinuclear endoplasmic reticulum (ER) region. A punctate expression pattern of Btz was also detected in the dendritic compartment (Fig. 6B). High magnifications of two representative dendrites (proximal-top to distal-bottom) are shown in Figure 6C. Interestingly, a nonhomogeneous pattern of colocalization was observed depending on the distance of the dendritic particle from the cell body. A quantitative analysis of the confocal images from three different neurons (Fig. 6D) revealed that an almost complete colocalization between $\mathrm{mBtz}$ and 
Staul was observed in the cell body segment $(>93 \pm 5.54 \%)$, in agreement with the nucleocytoplasmic shuttling activity of mBtz. A significant colocalization $(38 \pm 15.2 \%$; up to $70 \pm 17.86 \%)$ was still detected in the proximal dendritic compartment ( $\leq 1$ cell body in distance from the soma) that was decreasing in distal dendrites ( $32 \pm 17.4 \%$; up to $45 \pm 22.2 \%$ ) with increasing distance from the cell body. To prove that $\mathrm{mBtz}$ and Staufen 1 are indeed interacting in hippocampal neurons, we cotransfected neuronswith constructs expressing $\mathrm{mBtz}$ and Staufen1-HA (Fig. 6E). Again, both proteins were recruited into the same, discrete particular structures in the cell body as well as in dendrites (Fig. 6E). This suggests that both proteins are actually part of the same transport machinery.

\section{$\mathrm{mBtz}$ interacts with Staufen in an RNA-dependent manner}

To verify whether Staufen 1 and Btz interact, we performed immunoprecipitation experiments on brain extracts using two different anti-Staufen 1 polyclonal antibodies (Fig. 7A, S1 and S2). The immunoprecipitates obtained were then subjected to SDS-PAGE followed by Western blot. As shown in Figure 7A, Btz was specifically coimmunoprecipitated with Staufen1; no coimmunoprecipitation was observed whenthe preimmune (PI) serum was used instead. These results suggest that mBtz is a new component of dendritic RNPs.

To further investigate the specificity of the molecular interaction of $\mathrm{mBtz}$ and Staufen 1 in vivo, we performed coimmunoprecipitation assays from $\mathrm{BHK}$ cells cotransfected with tagged proteins. In detail, the following constructs coding for Staufen1-HA (Stau1-HA) and for either full-length Btz (Btz-GFP or untagged Btz) or truncated $\mathrm{Btz}\left(\mathrm{Btz}_{1-359}-\mathrm{GFP}\right)$ were used. The latter construct contains the $\mathrm{N}$-terminal part of $\mathrm{mBtz}$ including the homologous domain with Drosophila Btz (van Eeden et al., 2001) (Fig. 1, supplementary data). After cotransfection of BHK cells, IP assays were performed using either anti-GFP (Fig. 7B) or anti-HA antibodies (data not shown), and the immunoprecipitated proteins were visualized by Western blotting using anti-HA, antiGFP, anti-Btz, or anti c-myc antibodies (Fig. 7C). Stau1-HA interacted with both full-length Btz-GFP and the N-terminal $\mathrm{Btz}_{1-359}-\mathrm{GFP}$ fusion proteins indicating that both Staufen 1 and Btz are components of a common protein complex (Fig. 7B). Similar results were obtained when the coimmunoprecipitation was done using anti-HA antibodies (data not shown); this con-
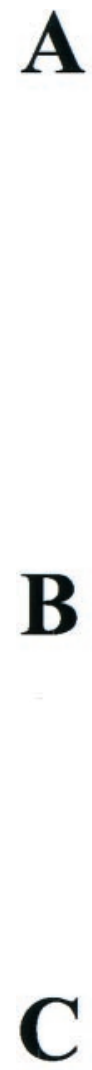

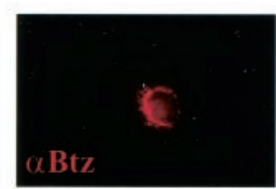

D
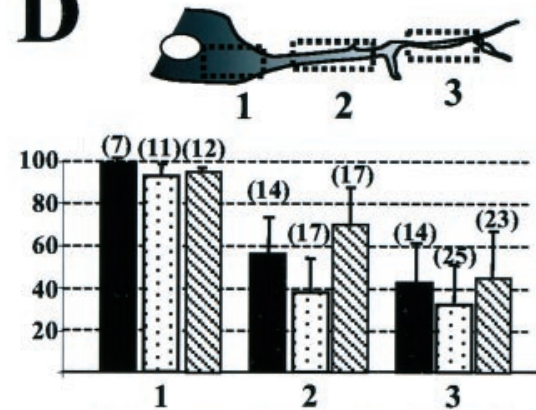

Cell body Proximal Distal
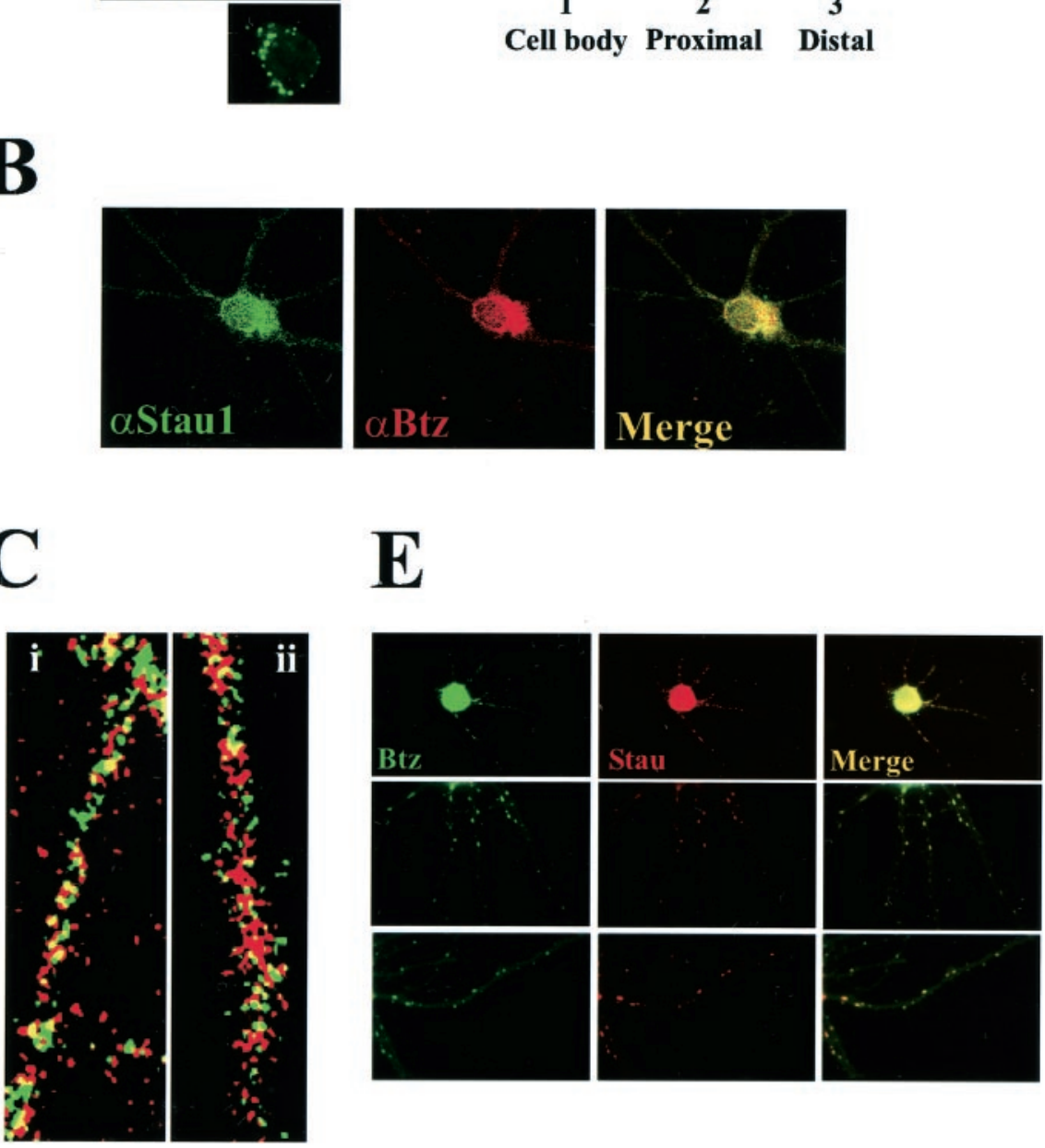

Figure 6. Intracellular localization of $\mathrm{mBtz}$ protein in hippocampal neurons. $A$, Endogenous $\mathrm{mBtz}$ and $\mathrm{mBtz}-\mathrm{GFP}$ show a remarkably similar localization in the cell body of primary hippocampal neurons (10-12 DIV). Neurons were immunostained for $\mathrm{mBtz}$ protein (top panel) or transiently transfected with a construct encoding $\mathrm{mBtz}-\mathrm{GFP}$, and fluorescent images were taken from a representative cell (bottom panel). Both proteins yielded a typical punctate, perinuclear localization (see also inset). $B$, Confocal images from fixed mature hippocampal neurons in culture. Like Staufen1, mBtz forms particles that localize to the dendritic compartment. The immunostaining was performed with affinity-purified rabbit polyclonal anti-mBtz antibodies. C, Higher magnifications of confocal microscopical images. Two different regions of dendrites (proximal-top to distal-bottom) are shown. Hippocampal neurons were labeled with immunopurified rabbit polyclonal anti-mBtz (green) and mouse anti-Stau1 (red). D, Quantitative analysis to evaluate the colocalization between $\mathrm{mBtz}$ and Stau1. Three different regions (shown in the schematic drawing) were chosen. The three-column set represents quantifications from three distinct neurons. The number on the top of each column indicates the number of areas analyzed per region. E, In vivo colocalization of Staufen 1 and $\mathrm{mBtz}$ in transfected hippocampal neurons. Mature neurons were transiently transfected with constructs encoding mBtz and Staufen1-HA, and then immunostaining was performed. Fluorescent images were taken from three different cells. Panels marked Merge in $B$ and $E$ are an overlay of the two images to the left. $\alpha$ Btz, Anti-Btz antibodies; $\alpha$ Stau1, anti-Staufen1 antibodies.

firmed that the order of IPs of both proteins was irrelevant in this assay. To investigate whether the observed interaction was specific for the two proteins analyzed, the following additional control IPs were performed. First, no coimmunoprecipitation was 
$\mathbf{A}$

\section{IP ( $\alpha$ Stau1)}

PI S1 S2

100

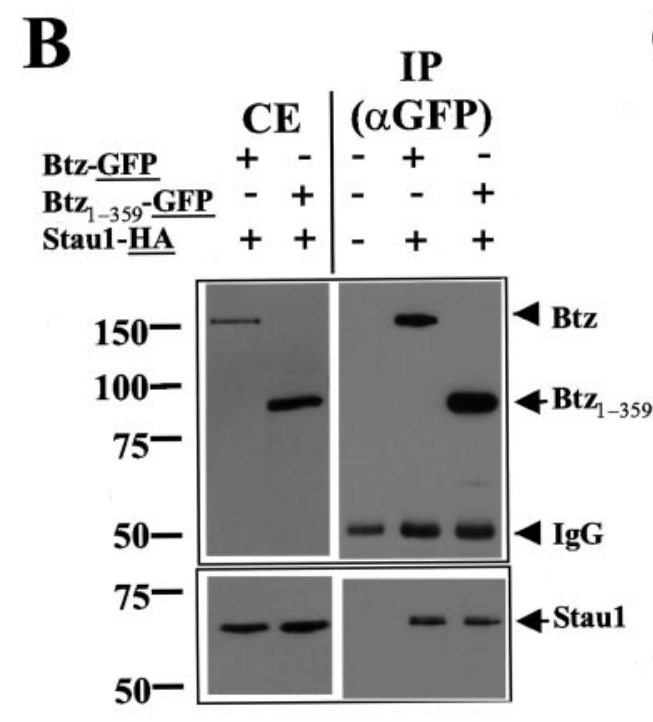

$\mathbf{C}$
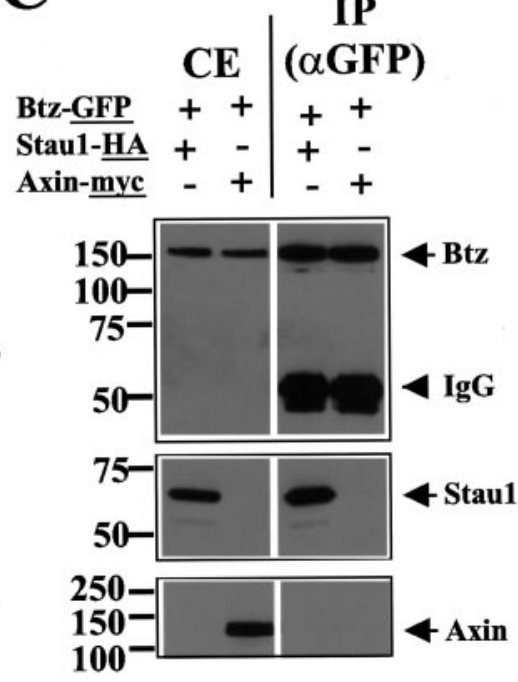

Figure 7. mBtz coimmunoprecipitates with Staufen1. A, Endogenous Staufen 1 immunoprecipitates Btz. IPs were performed with two different antisera $(S 1, S 2)$, and $\mathrm{mBtz}$ was detected with a specific antibody. As negative control, the IP was performed with the preimmune serum (PI). B, C, In vivo Staufen1-mBtz interaction. BHK cells were cotransfected with different constructs and lysed, and immunoprecipitations were performed as indicated. $B, B \mathrm{Btz}-\mathrm{GFP}$ and $\mathrm{Btz}_{1-359}-\mathrm{GFP}$ coimmunoprecipitate with Stau1HA. The left panel includes the indicated cell extracts (CE) showing the respective molecular masses of mBtz-GFP and Staufen1HA. IPs (right panel) were performed with anti-GFP antibodies ( $\alpha$ GFP), and proteins were visualized by Western blotting (underlined tags indicate the various antibodies). C, The interaction of $\mathrm{mBtz}$ is specific for Staufen1. Cotransfection of BHK cells with constructs expressing both mBtz-GFP and axin1-myc did not result in a coimmunoprecipitation of the two recombinant proteins. $\alpha$ Stau1, anti-Staufen1 antibodies.

observed when the pull-down was performed with monoclonal anti-reticulin antibodies (data not shown). Second, GFP alone did not pull down endogenous Staul (data not shown) or Staul-HA protein (Fig. 8B). Finally, when BHK cells were cotransfected with Btz-GFP and another overexpressed protein (e.g., axin1-myc instead of Stau1-HA), Btz-GFP specifically interacted with Staufen1-HA, but not with axin1-myc, in these coimmunoprecipitation assays (Fig. $7 C$ ). Together, these experiments indicate that Staufen 1 and Btz proteins specifically interact with each other in mammalian cells.

To investigate a possible role of $\mathrm{mBtz}$ in RNA transport and whether it is a component of Staufen-containing RNPs, we performed differential centrifugation of rat brain extract. The P100 and S100 derived from rat total brain were run on SDS-PAGE and blotted with anti-mammalian Btz antibodies. The majority of $\mathrm{mBtz}$ protein was associated with the pellet, indicating that it may be associated with intracellular membranes. Similar results have been obtained for Staufen proteins, because they are also primarily found in the P100-containing intracellular membranes and ribosomes (Ohashi et al., 2002; Mallardo et al., 2003). Interestingly, RNase treatment before differential centrifugation caused a significant release of both Staufen proteins as well as $\mathrm{mBtz}$ protein from the pellet into the $\mathrm{S} 100$ fraction (Fig. $8 \mathrm{~A}$ ). DNase treatment, in contrast, had no effect. These data strongly suggest that both Staufen 1 and $\mathrm{mBtz}$ proteins may be components of the same particles that are associated with intracellular membranes or ribosomes, most likely ER, and that RNase treatment releases these particles into the supernatant. Calnexin, a known ER marker, served as membrane marker (Ou et al., 1993).

We therefore asked the question whether the interaction of $\mathrm{mBtz}$ with Staufen1 in our coimmunoprecipitation assays might also be mediated by RNA. Interestingly, RNase treatment abolished Btz and Staufen1 interaction (Fig. 8B), confirming that RNA plays a role in the interaction. In contrast, DNase treatment did not significantly affect the Btz and Staufen1 interaction (data not shown). Because homology between Drosophila and mammalian Btz proteins is restricted to the N-terminal domain (Fig. 1, supplementary data), and the interaction of mBtz with Staufen1 appears to be conserved, we went on to analyze whether the $\mathrm{N}$-terminal conserved region of $\mathrm{mBtz}$ was indeed responsible for the interaction with Staufen1. Coimmunoprecipitation assays with Staufen1-HA and several $\mathrm{N}$-terminal domains of $\mathrm{mBtz}$ tagged with GFP (Fig. 8C) clearly showed that both $\mathrm{Btz}_{1-359}$ and $\mathrm{Btz}_{152-359}-\mathrm{GFP}$ fusion proteins still have the ability, as does fulllength Btz-GFP, to interact with Stau1-HA in an RNA-dependent manner. In contrast, the Btz fragment encompassing the first 158 aa $\left(\mathrm{Btz}_{1-158}-\mathrm{GFP}\right)$, which does not contain the homology domain, did not coimmunoprecipitate with Staufen 1 (Fig. 8C). Together, several conclusions can be drawn from these experiments. First, the interaction of $\mathrm{mBtz}$ with Staufen is mediated by the region homologous to Drosophila Btz, confirming that the interaction domain has been conserved during evolution. Second, our experiments suggest that mBtz selectively binds to Staufen 1 in the RNA-bound conformation and then possibly forms RNA-containing Staufen1 complexes in mammalian cells. To determine whether localized mRNAs were physically associated with the endogenous $\mathrm{mBtz}$ complex, as in the case of Staufen1 (Mallardo et al., 2003), we performed RT-PCR on immunoprecipitates from rat brain extracts using anti-mBtz antibodies. Primers specific for localized RNAs [e.g., BC1, MAP2, or $\mathrm{Ca}^{2+}$-calmodulin kinase $\mathrm{II} \alpha$ $(C a M K I I \alpha)$ (data not shown)] were used. As negative controls, primers were designed to detect both $\beta$-actin and Histone $\mathrm{H1}$ mRNAs (Fig. 9A). The IPs that were performed either in the absence of the primary immunopurified antibodies or in the presence of the preimmune rabbit serum (data not shown) did not reveal any PCR-amplified cDNA product (lane 1). In contrast, $B C 1$ transcript (lane 2) was clearly detected in the $\mathrm{mBtz}$ immunoprecipitates. In contrast, MAP2 was not detected (Fig. $9 A)$. Both $\beta$-actin and Histone $H 1$ transcripts were also not amplified from the immunoprecipitates (Fig. 9A). To check for the presence of additional mRNAs in the mBtz immunoprecipitates, radioactive RT-PCR was performed (Mallardo et al., 2003). The 


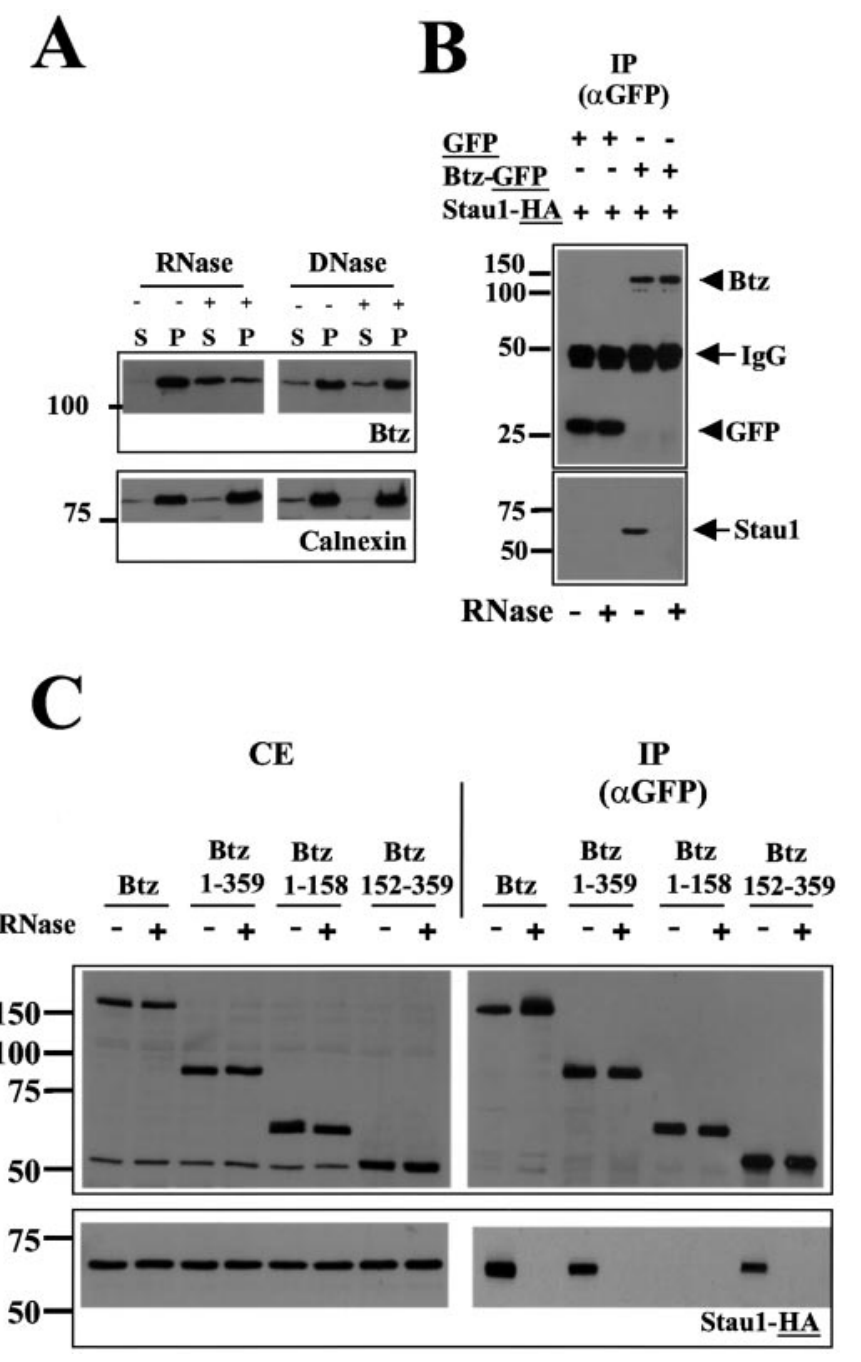

Figure 8. The interaction of Btz and Staufen 1 is RNA dependent. A, RNA-dependent interaction of $\mathrm{mBtz}$ with organelles. RNase treatment of $\mathrm{S} 14$ supernatants from 3-week-old rat forebrain extracts releases $\mathrm{mBtz}$ from the P100 (P) into the soluble fraction (S). DNase treatment, in contrast, does not change the distribution of $\mathrm{mBtz}$ protein. The same membrane was redecorated with monoclonal anti-calnexin antibodies [kind gift of Ari Helenius (Eidgenössische Technische Hochschule, Zürich, Switzerland)] to show that the ER marker calnexin is not removed by RNase treatment. $B$, mBtz interacts with Staufen 1 in an RNA-dependent manner. BHK cells were cotransfected and processed as indicated in Figure 7. Extracts were pretreated with RNase where indicated, and IPs were performed with anti-GFP antibodies. In this experiment, Btz-GFP and Stau1-HA only coimmunoprecipitate in the presence of RNA. Treatment with RNase A before the IP abolishes this interaction. As a control, GFP alone does not interact with Staufen 1 . The underlining indicates the portion of the antigen recognized by the antibodies. C, The conserved central domain of mammalian Btz is responsible for the RNA-dependent interaction with Staufen1. Different constructs coding for various parts of Btz were expressed together with Stau1-HA in BHK cells, and IPs were performed using anti-GFP antibodies in the presence or absence of RNase. The region from amino acid 152-359 in mouse Btz protein mediates the interaction with Stau1-HA. This domain contains the two identified motifs (1 and 2) (Fig. 1) that are conserved between Drosophila and mBtz proteins. CE, Cell extract; $\alpha \mathrm{GFP}$, Anti-GFP antibodies.

first two lanes of Figure $9 B$ represent mRNAs derived from S100 supernatants from either protein A-Sepharose alone (lane 1) or mBtz antibodies bound to protein A-Sepharose (lane 2). The observed pattern represents cytosolic mRNAs. In contrast, a distinct set of bands was detected in the Btz immunoprecipitate (Fig. $9 B$, lane 4 , arrowheads). The control lane 3 did not yield any band, indicating that no RNA binding protein binds to protein A-Sepharose. Whether these unknown bands actually represent

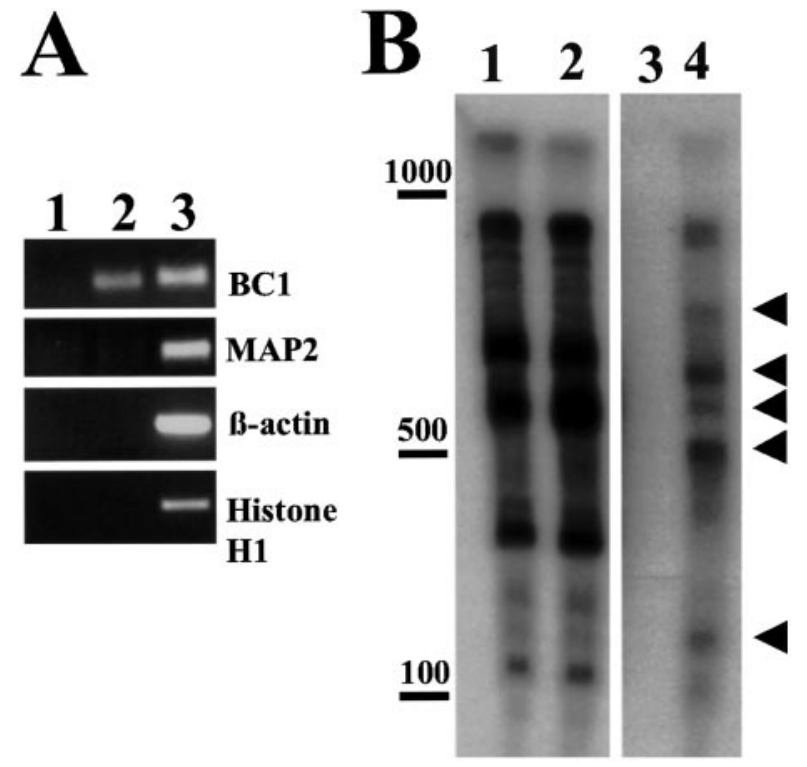

Figure 9. Characterization of RNA in $\mathrm{mBtz}$ immunoprecipitates. $A$, The dendritically localized $B C 1$ transcript is present in the $\mathrm{mBtz}$ IPs. RNA isolated from the mBtz IPs was retrotranscribed, and the resulting CDNA was amplified using primers specific for either dendritically localized (BC1, MAP2) or nonlocalized ( $\beta$-actin, Histone H1) transcripts. RNA was collected from IPs performed either in absence of antibodies (lane 1) or with immunopurified anti-mBtz polyclonal antibodies (lane 2 ) and then retrotranscribed, and the indicated CDNAs were detected by PCR. As positive controls, PCRs were also performed on CDNA from S100 (lane 3). B, Radioactive labeling of mRNAs present in the mBtz IPs. Radioactive RT-PCR was performed on RNA extracted from either mBtz immunoprecipitates (lane 4) or the corresponding supernatant (lane 2). As negative control, the anti-mBtz antibodies were omitted for the IPs (lane 3) to detect for mRNAs unspecifically bound to protein A-Sepharose beads. Lane 1 represents the corresponding supernatant. Arrowheads label a distinct set of bands that were specifically enriched in the mBtz IPs (lane 4).

potential cargo RNAs for the Btz-Staufen RNPs will be the subject of additional investigation.

\section{Discussion}

The purpose of this study was to identify additional proteins that interact with mammalian Staufen1. An attractive candidate for such an interactor is the mammalian homolog of Drosophila Btz, a protein component of the oskar RNPs that also contain Staufen, Mago nashi, Y14, and kinesin heavy chain (KHC) protein (van Eeden et al., 2001, and references therein). In btz1 homozygous mutants, these RNPs got stuck on their way to the posterior pole in the Drosophila oocyte. van Eeden et al. (2001) first pointed out the partial homology of Drosophila Btz with a previously identified mouse protein called MLN51. Its mRNA was found to be upregulated in cell lines derived from human metastatic breast and gastric cancer (Tomasetto et al., 1995; Varis et al., 2002). These facts prompted us to investigate whether MLN51 might represent a functional homolog of $\mathrm{mBtz}$ protein. Btz mRNA is highly expressed in the hippocampus and cerebellum where Staufen1 is also expressed (Monshausen et al., 2001; Duchaîne et al., 2002). When mBtz protein is made in the wild-type Drosophila egg chamber, it shows identical localization to the Drosophila protein in the female germline; Btz-GFP is detected in the perinuclear region of the nurse cells and localizes, together with Drosophila Staufen, to the posterior pole of the oocyte with the oskar mRNA localization complex. In btz mutant egg chambers, however, the mammalian protein can still enter the oocyte but remains at the anterior pole together with Staufen. This result is the first strong hint that MLN51 is the mammalian homolog of 
Btz protein, because it can still interact, through its conserved domain, with Drosophila Staufen. On the basis of these data, it is tempting to speculate that the apparent lack of ability to reach the posterior pole in the $b t z$ mutant oocyte is attributable to the difference within the $\mathrm{C}$ terminal of Btz. This $\mathrm{C}$-terminal region is not conserved in mammals, indicating a significant divergence during evolution.

In Drosophila, most Btz is associated with nuclear envelope membranes of the nurse cells and joins the oskar mRNA-Mago nashi protein complex as it is exported into the cytoplasm. In mammals, in contrast, Btz seems to behave as a nucleocytoplasmic shuttling protein. First, treatment with LMB, a drug that specifically inhibits CRM1-dependent protein export from the nucleus (Fukuda et al., 1997), led to the accumulation of BtzGFP in the nucleus. Second, the removal of either the two NLSs or the NES inhibited its cytosolic localization in mammalian fibroblasts. In addition to these sequence elements, there may be an additional domain at the very $\mathrm{N}$ terminal, located within the first $152 \mathrm{aa}$, of mammalian Btz protein that may be responsible for its cytoplasmic localization. Only if this domain is missing is the protein found exclusively in the nucleus of mammalian fibroblasts.

As in the case of the Drosophila Btz, the sequence of the mammalian homolog gave few clues to its biochemical functions. We therefore investigated whether mammalian Btz is still able to form a complex with mammalian Staufen1. In mammalian neurons, Staufen1-GFP forms particles in the cytoplasm that are associated with the nuclear-ER membrane system. Some of the smaller particles subsequently move in a saltatory manner along microtubules into dendrites of living hippocampal neurons (Köhrmann et al. 1999a). In this respect, it is of particular interest that both the endogenous and the recombinant Btz protein, as well as Staufen1, formed granules that can be found in the cell body and to a lesser extent in dendrites of hippocampal neurons. Most endogenous Btz protein, in comparison, not only localized in a ring shape around the nuclear membrane, but some staining was also detected in dendrites (Fig. 6). When we carefully evaluated the degree of colocalization between $\mathrm{mBtz}$ and Staufen1, we observed a somewhat dynamic pattern. Whereas the degree of colocalization was highest within the cell body, it gradually decreased with progressive distance of the particles from the cell body toward distal dendrites. There could be several reasons for the observed phenomenon. First, the interaction of $\mathrm{mBtz}$ with Staufen 1 might be highly dynamic and transient as in the case of Drosophila. Here, Drosophila Btz is only transiently associated with the oskar mRNA localization complex and is only found at the posterior pole when oskar mRNA localizes there (van Eeden et al., 2001). In the case of mature hippocampal neurons, this could indicate that $\mathrm{mBtz}$ only transiently associates with Staufen RNPs until they reach their final destination in dendrites. Therefore, the degree of colocalization progressively decreases because of the partial disassembly of $\mathrm{mBtz}$ from particles in dendrites. Alternatively, Btz could interact additionally with other double-stranded RNA binding proteins (e.g., Staufen2). Interestingly, a drastic increase in the degree of colocalization in Staufen1-containing particles was observed when both $\mathrm{mBtz}$ and Staufen 1 proteins were overexpressed in hippocampal neurons (Fig. 6E). This apparent difference between endogenous and overexpressed Btz protein could be explained by the fact that excess of both Staufen 1 and Btz may yield significantly larger particles that will then be subsequently transported into dendrites. The dynamics of the dendritic transport of Btz-containing particles is currently under investigation.

The mBtz-Staufen 1 interaction was then further validated by immunoprecipitations from doubly transfected BHK cells. These experiments showed that $\mathrm{mBtz}$ interacts with Staufen 1 through its conserved N-terminal domain. A thorough computer analysis revealed the existence of two conserved motifs within this domain, which are also present in other protein families. In this respect, it is of particular interest that $\mathrm{mBtz}$ protein does not share any other conserved domain, and that it is characterized by an unusual absence of any significant secondary structure (data not shown). Interestingly, mBtz and Staufen 1 do not seem to bind directly to each other, but this interaction appears to be mediated by RNA. This is in good accordance with the situation in Drosophila, in which both proteins assemble with oskar mRNA into RNPs (van Eeden et al., 2001). Preliminary evidence suggests that mBtz does not bind synthetic double-stranded RNA in an in vitro Northwestern assay (data not shown). Therefore, the RNAdependent association is mediated by either Staufen 1 or, alternatively, another RNA binding protein present in the complex. Additional work is required to identify the molecular nature of such additional RNA binding proteins present in Staufen-containing particles.

Unfortunately, the identity of the cargo RNA for mammalian Staufen proteins has not been revealed to date. A recent publication showed that Staufen1 binds to the dendritic targeting element of MAP2 mRNA in a trihybrid system (Monshausen et al., 2001). Whether this interaction actually occurs in vivo has not yet been demonstrated. Additional putative candidates for such cargo RNA (e.g., CaMKIIa, TrkB, and NMDAR1) have been characterized in a recent study by Krichevsky and Kosik (2001). The identification of cargo RNAs in Staufen RNPs, however, has been hampered by the fact that Staufen binds any double-stranded RNA in vitro (M. Kiebler, D. St. Johnston, L. DesGroseillers, unpublished observations). Therefore, we recently established a biochemical isolation procedure to enrich Staufen-containing RNPs from rat brain and to identify the bound RNA (Mallardo et al., 2003). This study showed that several dendritically localized RNAs are indeed enriched in the Staufen particles (e.g., the noncoding RNA BC1, CaMKII $\alpha$, as well as 15 novel RNAs) (M. Mallardo and M. Kiebler, unpublished observation). We succeeded in detecting one of these potential Staufen 1 cargo RNAs, $B C 1$, in our mBtz immunoprecipitates. Additionally, we present new evidence of the presence of a restricted number of novel, not-yet-characterized mRNAs that are associated with mBtz. The detection of BC1 RNA together with the presence of additional mRNAs within both Staufen 1 and mBtz complexes clearly strengthens the hypothesis that $\mathrm{mBtz}$ and Staufen may be part of the same particles, and that the observed interaction between these two proteins occurs in vivo. Additional work is needed, however, to characterize the RNAs that mediate the binding of Staufen 1 to Btz and to unravel the molecular mechanism of this interaction.

Together, our results suggest the following scenario. Btz is exported from the nucleus into the cytoplasm as a component of the ribonucleoprotein complex. Staufen 1 can then be recruited into this complex via its bound cargo RNA. Alternatively, the RNA-dependent interaction of mBtz with Staufen 1 could actually be mediated by another RNA binding protein that possibly exists within such an RNP (Wilhelm et al., 2000; Ohashi et al., 2002; Arn et al., 2003). This complex can then be transported into the dendritic compartment along microtubules with the help of KHC (S. Kroening, unpublished observations). 


\section{References}

Arn EA, Cha BJ, Theurkauf WE, Macdonald PM (2003) Recognition of a bicoid mRNA localization signal by a protein complex containing Swallow, Nod, and RNA binding proteins. Dev Cell 4:41-51.

Bashirullah A, Cooperstock RL, Lipshitz HD (1998) RNA localization in development. Annu Rev Biochem 67:335-394.

Bassell GJ, Oleynikov Y, Singer RH (1999) The travels of mRNAs through all cells large and small. FASEB J 13:447-454.

Degot S, Regnier CH, Wendling C, Chenard MP, Rio MC, Tomasetto C (2002) Metastatic Lymph Node 51, a novel nucleo-cytoplasmic protein overexpressed in breast cancer. Oncogene 21:4422-4434.

Duchaîne T, Wang HJ, Luo M, Steinberg SV, Nabi IR, DesGroseillers L (2000) A novel murine Staufen isoform modulates the RNA content of Staufen complexes. Mol Cell Biol 20:5592-5601.

Duchaîne TF, Hemraj I, Furic L, Deitinghoff A, Kiebler MA, DesGroseillers L (2002) Staufen2 isoforms localize to the somatodendritic domain of neurons and interact with different organelles. J Cell Sci 115:3285-3295.

Ephrussi A, Dickinson LK, Lehmann R (1991) Oskar organizes the germ plasm and directs localization of the posterior determinant nanos. Cell 66:37-50.

Erdelyi M, Michon AM, Guichet A, Glotzer JB, Ephrussi A (1995) Requirement for Drosophila cytoplasmic tropomyosin in oskar mRNA localization. Nature 377:524-527.

Fukuda M, Asano S, Nakamura T, Adachi M, Yoshida M, Yanagida M, Nishida E (1997) CRM1 is responsible for intracellular transport mediated by the nuclear export signal. Nature 390:308-311.

Kiebler MA, DesGroseillers L (2000) Molecular insights into mRNA transport and local translation in the mammalian nervous system. Neuron 25:19-28.

Kiebler MA, Hemraj I, Verkade P, Köhrmann M, Fortes P, Marión RM, Ortín J, Dotti CG (1999) The mammalian Staufen protein localizes to the somatodendritic domain of cultured hippocampal neurons: implications for its involvement in mRNA transport. J Neurosci 19:288-297.

Kim-Ha J, Smith JL, Macdonald PM (1991) oskar mRNA is localized to the posterior pole of the Drosophila oocyte. Cell 66:23-35.

Knoell B, Isenmann S, Kilic E, Walkenhorst J, Engel S, Wehinger J, Bähr M, Drescher U (2001) Graded expression patterns of ephrin-As in the superior colliculus after lesion of the adult mouse optic nerve. Mech Dev 106:119-127.

Köhrmann M, Luo M, Kaether C, DesGroseillers L, Dotti CG, Kiebler MA (1999a) Microtubule-dependent recruitment of Staufen-green fluorescent protein into large RNA-containing granules and subsequent dendritic transport in living hippocampal neurons. Mol Biol Cell 10:2945-2953.

Köhrmann M, Haubensak W, Hemraj I, Kaether C, Lessmann VJ, Kiebler MA (1999b) Fast, convenient, and effective method to transiently transfect primary hippocampal neurons. J Neurosci Res 58:831-835.

Krichevsky AM, Kosik KS (2001) Neuronal RNA granules: a link between RNA localization and stimulation-dependent translation. Neuron 32:683-696.

Macchi P, Hemraj I, Goetze B, Grunewald B, Mallardo M, Kiebler MA (2003) A GFP-based system to uncouple mRNA transport from translation in a single living neuron. Mol Biol Cell 14:1570-1582.

Mallardo M, Deitinghoff A, Müller J, Goetze B, Macchi P, Peters C, Kiebler MA (2003) Isolation and characterization of Staufen-containing ribonucleoprotein particles from rat brain. Proc Natl Acad Sci USA 100:2100-2105.
Micklem DR, Dasgupta R, Elliott H, Gergely F, Davidson C, Brand A, Gonzalez-Reyes A, St Johnston D (1997) The mago nashi gene is required for the polarisation of the oocyte and the formation of perpendicular axes in Drosophila. Curr Biol 7:468-478.

Monshausen M, Putz U, Rehbein M, Schweizer M, DesGroseillers L, Kuhl D, Richter D, Kindler S (2001) Two rat brain staufen isoforms differentially bind RNA. J Neurochem 76:155-165.

Monshausen M, Rehbein M, Richter D, Kindler S (2002) The RNA-binding protein Staufen from rat brain interacts with protein phosphatase-1. J Neurochem 81:557-564.

Newmark PA, Boswell RE (1994) The mago nashi locus encodes an essential product required for germ plasm assembly in Drosophila. Development 120:1303-1313

Ohashi S, Koike K, Omori A, Ichinose S, Ohara S, Kobayashi S, Sato TA, Anzai $\mathrm{K}$ (2002) Identification of mRNP complexes containing pur alpha, $\mathrm{mStaufen}$, fragile $\mathrm{X}$ protein and myosin $\mathrm{Va}$, and their association with rough endoplasmic reticulum equipped with a kinesin motor. J Biol Chem 277:37804-37810.

Ou WJ, Cameron PH, Thomas DY, Bergeron JJ (1993) Association of folding intermediates of glycoproteins with calnexin during protein maturation. Nature 364:771-776.

Palacios IM, St Johnston D (2001) Getting the message across: the intracellular localization of mRNAs in higher eukaryotes. Annu Rev Cell Dev Biol 17:569-614.

Palacios IM, St Johnston D (2002) Kinesin light chain-independent function of the Kinesin heavy chain in cytoplasmic streaming and posterior localisation in the Drosophila oocyte. Development 129:5473-5485.

Roegiers F (2003) Insights into mRNA transport in neurons. Proc Natl Acad Sci USA 100:1465-1466.

Schuler GD, Altschul SF, Lipman DJ (1991) A workbench for multiple alignment construction and analysis. Proteins 9:180-190.

Smith WB, Aakalu G, Schuman EM (2001) Local protein synthesis in neurons. Curr Biol 11:R901-R903.

St Johnston D, Beuchle D, Nüsslein-Volhard C (1991) Staufen, a gene required to localize maternal RNAs in the Drosophila egg. Cell 66:51-63.

Steward O, Schuman EM (2001) Protein synthesis at synaptic sites on dendrites. Annu Rev Neurosci 24:299-325.

Tang SJ, Meulemans D, Vazquez L, Colaco N, Schuman E (2001) A role for a rat homolog of Staufen in the transport of RNA to neuronal dendrites. Neuron 32:463-475.

Tetzlaff MT, Jaeckle H, Pankratz MJ (1996) Lack of Drosophila cytoskeletal tropomyosin affects head morphogenesis and the accumulation of oskar mRNA required for germ cell formation. EMBO J 15:1247-1254.

Tomasetto C, Regnier C, Moog-Lutz C, Mattei MG, Chenard MP, Lidereau R, Basset P, Rio MC (1995) Identification of four novel human genes amplified and overexpressed in breast carcinoma and localized to the q11q21.3 region of chromosome 17. Genomics 28:367-376.

van Eeden FJ, Palacios IM, Petronczki M, Weston MJ, St Johnston D (2001) Barentsz is essential for the posterior localization of oskar mRNA and colocalizes with it to the posterior pole. J Cell Biol 154:511-523.

Varis A, Wolf M, Monni O, Vakkari ML, Kokkola A, Moskaluk C, Frierson Jr H, Powell SM, Knuutila S, Kallioniemi A, El-Rifai W (2002) Targets of gene amplification and overexpression at $17 \mathrm{q}$ in gastric cancer. Cancer Res 62:2625-2629.

Wilhelm JE, Mansfield J, Hom-Booher N, Wang S, Turck CW, Hazelrigg T, Vale RD (2000) Isolation of a ribonucleoprotein complex involved in mRNA localization in Drosophila oocytes. J Cell Biol 148:427-440. 